\title{
FACTORIZATION OF GROUPS INVOLVING SYMMETRIC AND ALTERNATING GROUPS
}

\author{
M. R. DARAFSHEH and G. R. REZAEEZADEH
}

(Received 16 August 2000 and in revised form 28 September 2000)

\begin{abstract}
We obtain the structure of finite groups of the form $G=A B$ where $B$ is a group isomorphic to the symmetric group on $n$ letters $S_{n}, n \geq 5$ and $A$ is a group isomorphic to the alternating group on 6 letters.
\end{abstract}

2000 Mathematics Subject Classification. 20D40.

1. Introduction. For a finite group $G$ if there exist subgroups $A$ and $B$ of $G$ such that $G=A B$, then $G$ is called a factorizable group. Of course if neither of $A$ nor $B$ is contained in the other, then the factorization is called nontrivial. A knowledge of the factorizations of finite simple groups will help to investigate the general theory of factorizing finite groups. All possible factorizations of sporadic simple groups have been obtained in [4] and those of simple groups of Lie type of Lie rank 1 or 2 in [3].

A factorization $G=A B$ where both $A$ and $B$ are maximal subgroups of $G$ is called a maximal factorization of $G$. In [9], all the maximal factorizations of all the finite simple groups and their automorphism groups have been determined completely.

In another direction some results have been obtained assuming $G=A B$ is a factorization of $G$ with $A$ and $B$ simple subgroups of $G$. For example, in [8] finite groups $G=A B$ where both $A$ and $B$ are isomorphic to the simple group of order 60 are classified, and in [10] finite groups $G=A B$ where $A$ is a non-abelian simple group and $B \cong A_{5}$ are determined. In [5], $G=A B$ where $A$ and $B$ are simple groups of small order are considered.

In a series of papers, Walls considered groups which are a product of simple groups $[13,14]$. In [15], groups which are product of a symmetric group and a group isomorphic to $A_{5}$ are classified. This result is interesting because in the factorization $G=A B$ one of the factors is not a simple group. Motivated by this result, in this paper we classify all groups $G$ which are product of subgroups $A$ and $B$ such that $A \cong A_{6}$ and $B \cong S_{n}, n \geq 6$. In this paper, $A_{n}$ and $S_{n}$ are the alternating and symmetric groups on $n$ letters, respectively, and all groups are assumed to be finite.

2. Preliminary results. Now $A_{6}$ is a simple group of order 360 and it is easy to verify that the order of any proper subgroup of $A_{6}$ is one of the numbers $1,2,3,4,5$, $6,8,9,10,12,18,24,36$, or 60 . Therefore the size of sets on which $A_{6}$ acts transitively and faithfully is one of the numbers $360,180,120,90,72,60,45,40,36,30,20,15$, 10 , or 6 . Also since $A_{6} \cong L_{2}(9), A_{6}$ has a 2 -transitive action on a set of 10 points and by 
consulting [10] one can see that if $A_{6}$ acts $k$-transitively, $k \geq 2$, on a set of cardinality $m$, then either $m=10, k=2$ or $m=6$ and $k=2,3$, or 4 . Now by [14, Lemma 7] we have the following decomposition.

LEMMA 2.1. For $n$ a positive integer, $S_{n+1}=A_{6} S_{n}$ and $A_{n+1}=A_{6} A_{n}$ if and only if $n=5,9,14,19,29,35,39,44,59,71,89,119,179$, or 359 . We can write $A_{10}=A B$ where $A \cong A_{6}$ and $B \cong A_{8}$. Further, we can write $S_{10}=A B$ and $A_{10} \times Z_{2}=A B$ where $A \cong A_{6}$ and $B \cong S_{8}$.

The only nontrivial decomposition $A_{m}=A B$, where $A \cong A_{6}$ and $B \cong A_{n}$, occurs if and only if $m=n+1$, where $n$ is one of the numbers mentioned in Lemma 2.1 or $m=10$ and $n=8$. To see this one can use [14, Theorem 9]. Because according to this theorem one of the groups in the decomposition $G=A B$, say $A$, must be a $k$ transitive permutation group and according to what we said earlier all the $k$-transitive permutation representations of $A \cong A_{6}$ are known.

For our work it is necessary to know if it is possible to decompose an alternating group as the product of $A_{6}$ and $S_{n}$.

LEMмA 2.2. It is not possible to decompose the alternating group $A_{m}, m \geq 7$, as the product of $A_{6}$ and a symmetric group $S_{n}, n>1$, unless $m=10$ and $n=8$.

Proof. According to [9, Theorem D], if $A_{m}$ acts naturally on a set $\Omega$ of cardinality $m$, and $A_{m}=A_{6} S_{n}$, then there are two possibilities.

CASE (i). $A_{m-k} \unlhd S_{n} \leq S_{m-k} \times S_{k}$ for some $k, 1 \leq k \leq 5$, and $A_{6}$ is $k$-homogeneous on $\Omega$. If $k=1$, then $A_{m-1} \unlhd S_{n} \leq S_{m-1}$ and it is easy to deduce $n=m-1$. Therefore $A_{n+1}=A_{6} S_{n}$ and so, $S_{n} \leq A_{n+1}$ from which it follows that $n=1$ which is not the case. If $k \geq 2$ then by [7] $A_{6}$ can only be $k$-transitive for $k=2,3$, or 4 . If $k=2$, then $m=6$ or 10 . Since we have assumed that $m \geq 7$, therefore, if $m=10$, then $A_{10}=A_{6} S_{n}$ and from $A_{8} \unlhd S_{n} \leq S_{8} \times S_{2}$ we obtain $n \geq 8$ and the order consideration in $A_{10}=A_{6} S_{n}$ leads to $A_{10}=A_{6} S_{8}$. If $k=3$ or 4 , then $m=6$ and again $A_{6}=A_{6} S_{n}$, a contradiction. Since in [9, Theorem D] the role of $S_{n}$ and $A_{6}$ may be interchanged, hence we may assume that $A_{m-k} \unlhd A_{6} \leq S_{m-k} \times S_{k}$ and $S_{n}$ is $k$-homogeneous for some $1 \leq k \leq 5$. However, a contradiction is obtained in this case again.

CASE (ii). $m=6,8$, or 10 . If $m=6$ then $A_{6}=A_{6} S_{n}$, a contradiction. If $m=8$, then $A_{8}=A_{6} S_{n}$ from which it follows that $n \geq 7$, but it is easy to see that $A_{8}$ has no subgroup isomorphic to $S_{7}$. If $m=10$, then $A_{10}=A_{6} S_{n}$ from which it follows that $n=7$ or 8 .

Now to rule out the case $n=7$. We will use [16, Result 1.4]. Using the notation used in [16] the decomposition $A_{10}=A_{6} S_{7}$ is exact and we have $p=7$ and $|\Delta|=k=3$ and therefore $A_{6}$ must be 3 -homogeneous which is impossible by [7] unless $A_{6}$ acts on $\Omega,|\Omega|=6$ in a natural way and this is also a contradiction. However, if $n=8$, then $A_{10}=A_{6} S_{8}$ and this possibility holds because by Lemma 2.1 we have $A_{10}=A_{6} A_{8}$ and since $A_{10}$ has a subgroup isomorphic to $S_{8}$, namely $A_{8}\left\langle\left(\begin{array}{lll}1 & 2\end{array}\right)\left(\begin{array}{ll}9 & 10\end{array}\right)\right\rangle$ we obtain $A_{10}=A_{6} S_{8}$.

In this paper we also use the following result which can be proved using the subgroup structure of $L_{2}(q)$ given in [6]. 
TABLE 2.1. Primitive groups of degree $k \geq 5, k \mid 360$.

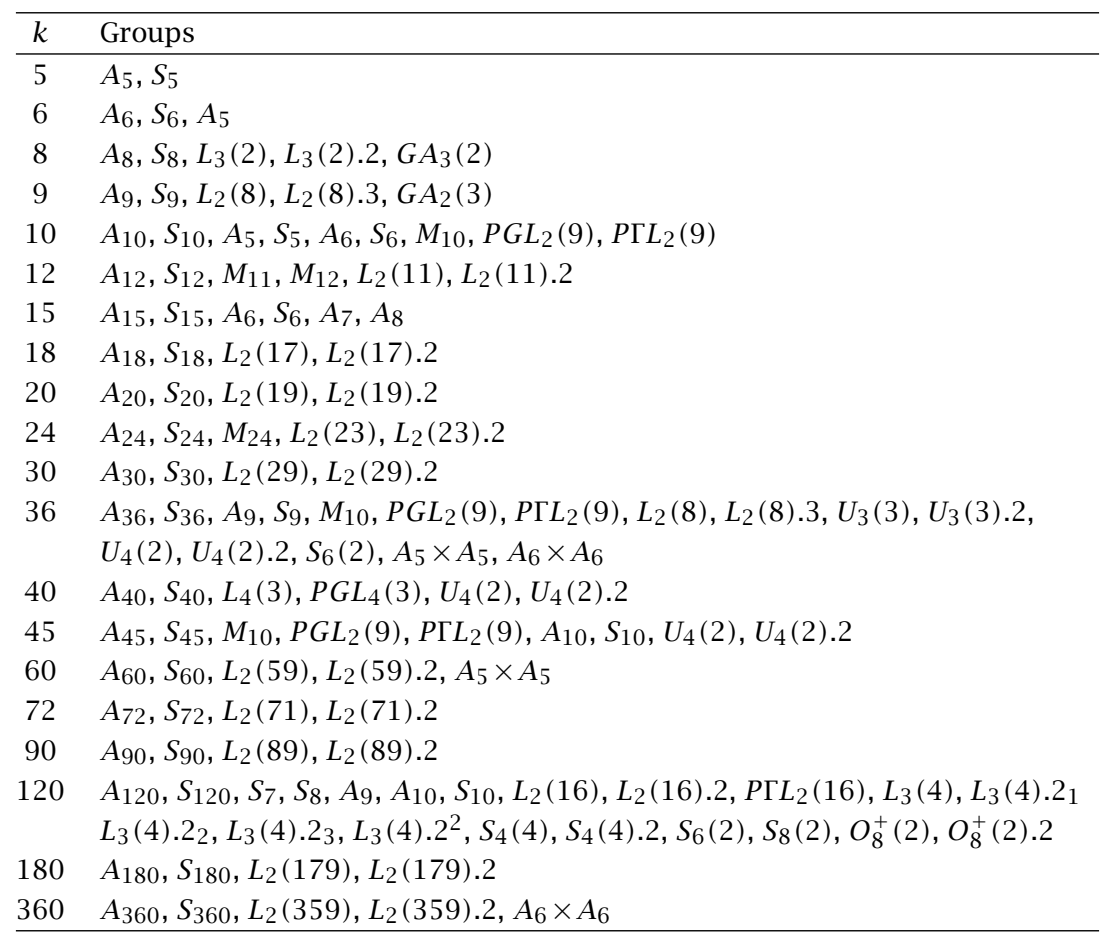

LEMMA 2.3. It is not possible to decompose the group $L_{2}(q)$ as the product of $A_{6}$ and $S_{n}$, where $n>4$.

Proof. By [6, page 213] if $G=L_{2}(q), q=p^{f}$, has a subgroup isomorphic to $A_{6}$, then this subgroup must be of the form $L_{2}\left(p^{m}\right)$ where $m \mid f$. But $L_{2}\left(p^{m}\right) \cong A_{6}$ if and only if $p=3$ and $m=2$, hence $G=L_{2}\left(3^{2 k}\right), k \geq 1$. But again by [6] a symmetric group $S_{n}$ can be a subgroup of $G$ if and only if $n \leq 4$, a contradiction.

[15, Lemma 3] is essential in this paper and so we will reproduce it here. We mention that it is not necessary to assume that $B$ is a complete group and our rephrasing of the lemma is as follows.

LEMmA 2.4. Suppose $G=A B$ is such that $A$ is a simple group and $B$ has a unique proper normal subgroup $N$ which is simple. Let $G \supsetneqq A \times B$ and let $M$ be a minimal normal subgroup of $G$. Then one of the following holds:

(i) $G=A B=M$ is a simple group

(ii) $G=M B, M=A \times N, N \cong A$

(iii) $G=M B, M \cong N A$ is simple

(iv) $M=A$ or $N,[G: A N]=[B: N], A N \cong A \times N$

(v) $M \cap X=1,|M| \mid[X: A \cap B]$ for $X \in\{A, B\},|M||A \cap B=| A M / M \cap B M / M \mid$.

Our work also depends on the primitive groups of certain degrees. Primitive groups of degree up to 20 were obtained in [11] and up to 1000 in [2]. In Table 2.1, the list 
of all primitive groups of degree $k \geq 5$ where $k$ is a divisor of $\left|A_{6}\right|=360$ is given. Notation for the names of groups in Table 2.1 is taken from [1].

3. Main results. In this section, using Lemma 2.4, we characterize finite groups $G=A B$ where $A \cong A_{6}$ and $B \cong S_{n}, n \geq 5$. But first we deal with the possibilities which arise as different cases in Lemma 2.4 .

LEMmA 3.1. There is no simple group $M$ such that $M=A B$ where $A \cong A_{6}$ and $B \cong S_{n}$, $n \geq 5$, unless $M \cong A_{10}$ and $n=8$.

Proof. We will assume that $M$ is a simple group having subgroups $A \cong A_{6}$ and $B \cong S_{n}, n \geq 5$, such that $M=A B$ and derive a contradiction. If $C$ is a maximal subgroup of $G$ containing $B$, then $M=A C$ and $k=[M: C]=[A: A \cap C] \mid 360$. Therefore $M$ is a primitive simple group of degree $k$, where $k$ is a divisor of 360 and $k \geq 5$. By Lemmas 2.2 and 2.3, we know that $M$ cannot be isomorphic to an alternating group or a linear group $L_{2}(g)$, unless $M \cong A_{10}$ for which the decomposition $A_{10}=A_{6} S_{8}$ is possible by Lemma 2.2.

Therefore by Table 2.1 we have the following possibilities for $M: M_{11}, M_{12}, M_{24}$, $U_{3}(3), U_{4}(2), L_{4}(3), L_{3}(4), S_{4}(4), S_{6}(2), S_{8}(2), O_{8}^{+}(2)$. Since $5 \nmid\left|U_{3}(3)\right|$, therefore $M=U_{3}(3)$ is impossible. If $M=M_{11}$ or $M_{12}$ then 11||$M \mid$ and hence $n \geq 11$ which implies that 7||$M \mid$ a contradiction. If $M=M_{24}$, then 23||$M \mid$ and so $n \geq 23$ implying that 17||$M \mid$, a contradiction. The same reasoning rules out $M=L_{4}(3), S_{4}(4)$ and $S_{8}(2)$ considering 13||$M \mid$ in the first case and 17||$M \mid$ in the remaining two cases. If $M=U_{4}(2)$, then as $\left|U_{4}(2)\right|=2^{6} \cdot 3^{4} \cdot 5=\left|A_{6} S_{n}\right|$ we must have $n=6$ and therefore $U_{4}(2)=A_{6} S_{6}$, but by [1] $U_{4}(2)$ has only one conjugacy class of subgroups isomorphic to $S_{6}$ and hence by [9, Proposition C, page 31] there is $g \in U_{4}(2)$ such that $U_{4}(2)=S_{6}^{g} S_{6}$ which by [12, page 26] is impossible. If $M=L_{3}(4)=A_{6} S_{n}$, then $n \geq 7$ but by [1] the group $L_{3}(4)$ has no subgroup isomorphic to $S_{7}$. If $M=S_{6}(2)=A_{6} S_{n}$, then as $\left|S_{6}(2)\right|=2^{9} \cdot 3^{4} \cdot 5 \cdot 7$ we obtain $7 \leq n \leq 10$ and since by [1] the group $S_{6}$ (2) has no subgroup isomorphic to $S_{9}$ hence $n=7$ or 8 . But order consideration yields $n=8$ and so $S_{6}(2)=A_{6} S_{8}$. By [1], the group $A_{6}$ cannot be contained in a maximal subgroup of the form $2^{5}: S_{6}$. Again by [1], the group $S_{6}(2)$ has only one conjugacy class of subgroups isomorphic to $S_{8}$ and so $S_{6}(2)=S_{8}^{g} S_{8}$ for some $g \in S_{6}$ (2) which is impossible by [12, page 26]. Finally, if $M=A_{6} S_{n}=O_{8}^{+}(2)$, then by [1] $n \leq 8$ and order consideration gives a contradiction.

LEMmA 3.2. Let $G$ be a group such that $G=A B$ where $A \cong A_{6}$ and $B \cong A_{n}, n \geq 5$, then either $G \cong A \times B$ or one of the following cases holds:

(i) $G=A_{n+1}, n=5,9,14,19,29,35,39,44,59,71,89,119,179$, or 359

(ii) $G=A_{n}, n \geq 6$, or

(iii) $G=A_{10}, n=8$.

Proof. First suppose that $G$ is simple. By Lemma 2.1 the cases (i) and (iii) are possible and the case (ii) arise from the trivial factorization of $A_{n}$. Now assume that the simple group $G$ has the desired decomposition $G=A_{6} A_{n}, n \geq 5$ and let $C$ be a maximal subgroup of $G$ containing $A_{n}$. Therefore $G=A_{6} C$ and $m=[G: C]=\left[A_{6}: A_{6} \cap C\right] \mid 360$. 
Maximality of $C$ in $G$ implies that $G$ is a simple primitive permutation group of degree $m$ where $m$ is a divisor of 360 . Now by Table 2.1 we know that simple primitive permutation groups are alternating groups, sporadic simple groups and simple groups of Lie type with small orders. We consider the following cases:

(a) The group $G$ is isomorphic to an alternating group. In this case by Lemma 2.1 and what follows after that we obtain all the cases (i), (ii), and (iii) of the lemma.

(b) The group $G \cong L_{2}(q)$ is a 2-dimensional linear group over the finite field $G F(q)$. In this case by [3] factorization $L_{2}(q)=A_{6} A_{n}$ is possible if and only if $n=6$ and $q=9$ which gives the trivial factorization.

(c) The group $G$ is isomorphic to a sporadic simple group. In this case by Table 2.1 we have the following possibilities for $G=M_{11}, M_{12}, M_{24}$. But by [4] the factorization $G=A_{6} S_{n}, n \geq 5$, is not possible for $G$.

(d) The group $G$ is isomorphic to one of the following linear groups:

$U_{3}(3), U_{4}(2), L_{4}(3), L_{3}(4), S_{4}(4), S_{6}(2), S_{8}(2), O_{8}^{+}(2)$. Since $5 \nmid\left|U_{3}(3)\right|$ therefore $G \neq U_{3}(3)$. If $G=S_{4}(4)$ or $S_{8}(2)$, then since 17||$G \mid$ we must have $n \geq 17$ and since $13 \nmid|G|$ we get a contradiction. If $G=L_{4}(3)$, then 13||$G \mid$ and so $n \geq 13$ which is impossible because $11 \nmid|G|$. If $G=U_{4}(2)=A_{6} A_{n}$, then order consideration yields $n=6$. But by [1] the maximal subgroup of $U_{4}(2)$ containing one of the $A_{6}$ subgroups is conjugate to an $S_{6}$ subgroup which is maximal in $G$. Therefore $U_{4}(2)=A_{6} S_{6}$ which is impossible by the proof of Lemma 3.1. If $G=L_{3}(4)=A_{6} A_{n}$, then by [1] $n=6$, a contradiction because 7||$G \mid$. If $G=S_{6}(2)=A_{6} A_{n}$ then since $3^{4}|| G \mid$ and $S_{6}(2)$ has no subgroup isomorphic to $A_{9}$ we must have $n=8$. But $G=A_{6} A_{8}$ and $A_{8}$ is contained in a maximal subgroup of $S_{6}(2)$ isomorphic to $S_{8}$ and so $G=A_{6} S_{8}$ which is impossible by Lemma 3.1. Finally if $G=O_{8}^{+}(2)=A_{6} A_{n}$, then by [1] $n \leq 9$ and order consideration gives a contradiction.

Now suppose that $G$ is not isomorphic to $A \times B$ and let $1 \neq M$ be a minimal normal subgroup of $G$. By [14, Lemma 1] $M$ is elementary abelian, $M \cap A=M \cap B=1$, and $|M|$ divides 360 the order of $A_{6}$. Thus $M$ is an elementary abelian subgroup of order $2,2^{2}, 2^{3}, 3,3^{2}$, or 5 . By induction, as $G / M=(A M / M)(B M / M)$ with $A M / M \cong A$ and $B M / M \cong B$, that $G / M$ is simple. Hence, either $C_{G}^{(M)}=M$ or $M \leq Z(G)$. Now $C_{G}^{(M)}=M$ implies that $A_{6} \leq \operatorname{Aut}(M)$, contrary to the possibilities for $M$. Now $M=Z(G)$ and $G / M$ is an alternating group. It follows that $G$ is a covering group of an alternating group, contrary to [14, Theorem 10].

THEOREM 3.3. Let $G$ be a group such that $G=A B, A \cong A_{6}$ and $B \cong S_{n}, n \geq 5$. Then one of the following cases occurs:

(a) $G \cong A_{6} \times S_{n}$

(b) $G \cong A_{10} \cong A_{6} S_{8}, n=8$

(c) $G \cong\left(A_{6} \times A_{6}\right)\langle\tau\rangle, \tau$ an automorphism of order 2 and $A_{6} \times A_{6}$ is the minimal normal subgroup of $G, n=6$

(d) $G \cong S_{n+1}, n=5,9,14,19,29,35,39,44,59,71,89,119,179,359$

(e) $G \cong S_{n}, n \geq 6$

(f) $G \cong A_{10} \times Z_{2}, n=8$

(g) $G \cong\left(A_{6} \times A_{n}\right)\langle\tau\rangle, n \geq 5$, where $\tau$ acts as an automorphism of order 2 on both factors. 
Proof. Our proof is based on the results of Lemma 2.4 and here we use the same notation used in this lemma. Therefore, let $M$ be a minimal normal subgroup of $G$ and note that $N \cong A_{n}$. If $G \supsetneqq A \times B$, then one of the following possibilities occurs:

(1) $M=G=A B$ is a simple group. In this case by Lemma 3.1 we have $M \cong A_{10}$ and $n=8$ and case (b) occurs.

(2) $G=M B, M \cong A_{6} \times N, N \cong A_{6}$.

In this case $n=6$ and $G \cong A_{6} S_{6}, S_{6}$ acts on $A_{6}$ by conjugation and $A_{6} \times A_{6}$ is the minimal normal subgroup of $G$ and this is case (c) in the theorem.

(3) $G=M B, M \cong A_{6} A_{n}$ is simple. In this case by Lemma 3.2 three cases occur. If $M=A_{n+1}, n=5,9,14,19,29,35,39,44,59,71,89,119,179$, or 359 , then the same reasoning used in the proof of [15, Theorem 4] yields case (d). If $M=A_{n}$, then $G=S_{n}$, $n \geq 6$ and this is the case (e). If $M=A_{10}$ and $n=8$, then a simple argument forces $G \cong S_{10}$ or $A_{10} \times Z_{2}$. If $G \cong S_{10}$ we have case (e) again. If we consider the alternating group $A_{10}$ on the set $\{1,2, \ldots, 10\}$. Then since $A_{6}$ has a 2-transitive action on 10 letters we obtain $A_{10}=A_{6} A_{8}$ where $A_{8}$ is the pointwise stabilizer of the set $\{9,10\}$. Now the set stabilizer of $\{9,10\}$ is isomorphic to $S_{8}$ and is a subgroup of $A_{10}$ containing this $A_{8}$. Therefore $A_{10}\left\langle\left(\begin{array}{ll}9 & 10\end{array}\right)\right\rangle=A_{6} A_{8}\left\langle\left(\begin{array}{ll}9 & 10\end{array}\right)\right\rangle$ implying $A_{10} \times Z_{2} \cong A_{6} S_{8}$ which is the case (f).

(4) $M=A_{6}$ or $A_{n},\left[G: A_{6} A_{n}\right]=2, A_{6} A_{n} \cong A_{6} \times A_{n}$. In this case $G \cong\left(A_{6} \times A_{n}\right) \cdot\langle\tau\rangle$ where $\tau$ acts as an outer automorphism of order 2 on both factors and this is the case (g).

(5) $M \cap A=1, M \cap B=1$ and $|M|$ divides $\left|A_{6}\right|$.

Since $M$ is isomorphic to a direct product of simple groups either $M$ is isomorphic to $A_{6}, A_{5}$ or $M$ is elementary abelian of order $2,2^{2}, 2^{3}, 3,3^{2}$, or 5 . If $M \cong A_{6}$, then as $M S_{n} \leq$ $G$ and $M \cap B=1, G=M B \cong A_{6} S_{n}$ with $A_{6}$ as a minimal normal subgroup. This is the case (4) treated above. Consider $C_{G}^{(M)}$. Suppose that $A \cap C_{G}^{(M)}=1$. Then $A$ is isomorphic to a subgroup of $\operatorname{Aut}(M)$. Considering the possibilities for $M$, this is impossible. Thus, $A \leq C_{G}^{(M)}$ and by the modular law $C_{G}^{(M)}=A\left(B \cap C_{G}^{(M)}\right)$. Now since $B \cap C_{G}^{(M)}$ is a normal subgroup of $B$, we must have either $B \cap C_{G}^{(M)}=1, B$, or $B \cap C_{G}^{(M)} \cong A_{n}$. If $B \cap C_{G}^{(M)}=1$, then as before $B$ is isomorphic to a subgroup of $\operatorname{Aut}(M)$, contrary to the possibilities for $M$ unless $M \cong A_{5}$ and $n=5$. Now $A M$ has index 2 in $G$, so $A M=C_{G}^{(M)} \times M$ is a normal subgroup of $G$. This is case (4), above. If $B \cap C_{G}^{(M)} \cong A_{n}$, then $C_{G}^{(M)}$ is as in Lemma 3.2. However, none of these groups has a nontrivial center, a contradiction. Thus, we must have $B \leq C_{G}^{(M)}$ and $M \leq Z(G)$ and hence, $M$ has prime order. By induction, $G / M=$ $(A M / M)(B M / M)$ must be in the list, but $(A M / M) \cap(B M / M) \neq 1$ so only the parts (b), (d), (e), and (f) are possible. If part (e) holds, then we would have $G=B M=B \times M$ contrary to the fact that $A$ has no subgroup of prime index. If part (b) or (d) holds, then $G$ is the covering group of the symmetric group. Now we can see that $B M / M$ must contain an involution which is the product of 2-cycles. It is known, see [10], that such an involution must lift to an element of order 4 in $G$, contrary to the fact that $M \cap B=1$. (Note that $B M / M$ lifts to $B M$ in $G$, see the argument in [14].) Now suppose that $G / M=A_{10} \times Z_{2}$ and $n=8$. Thus $G$ has a normal subgroup of order $2|M|$ which arguing as above must be the center of $G$. It follows that $G$ is a covering group of $A_{10}$. But as the Schur multiplier of $A_{10}$ has order 2 this is impossible. This completes the proof. 
ACKNOWLEDGements. We would like to thank Professor Gary L. Walls for his interest and guidance in preparation of this manuscript. We also wish to thank the referee for his comments concerning improvements in proving Lemma 3.2 and Theorem 3.3.

\section{REFERENCES}

[1] J. H. Conway, R. T. Curtis, S. P. Norton, R. A. Parker, and R. A. Wilson, Atlas of Finite Groups. Maximal Subgroups and Ordinary Characters for Simple Groups, Oxford University Press, Eynsham, 1985. MR 88g:20025. Zbl 568.20001.

[2] J. D. Dixon and B. Mortimer, The primitive permutation groups of degree less than 1000 , Math. Proc. Cambridge Philos. Soc. 103 (1988), no. 2, 213-238. MR 89b:20014. Zbl 646.20003.

[3] T. R. Gentchev, Factorizations of the groups of Lie type of Lie rank 1 or 2, Arch. Math. (Basel) 47 (1986), no. 6, 493-499. MR 87k:20033. Zbl 589.20006.

[4] _ Factorizations of the sporadic simple groups, Arch. Math. (Basel) 47 (1986), no. 2, 97-102. MR 88f:20031. Zbl 591.20022.

[5] H. Hanes, K. Olson, and W. R. Scott, Products of simple groups, J. Algebra 36 (1975), no. 2, 167-184. MR 55\#10558. Zbl 311.20007.

[6] B. Huppert, Endliche Gruppen I, Die Grundlehren der Mathematischen Wissenschaften, vol. 134, Springer-Verlag, Berlin, 1967 (German). MR 37\#302. Zbl 217.07201.

[7] W. M. Kantor, k-homogeneous groups, Math. Z. 124 (1972), 261-265. MR 46\#5422. Zbl 232.20003.

[8] O. H. Kegel and H. Lüneburg, Über die kleine reidemeisterbedingung II, Arch. Math. 14 (1963), 7-10 (German). MR 26\#4253. Zbl 108.16302.

[9] M. W. Liebeck, C. E. Praeger, and J. Saxl, The maximal factorizations of the finite simple groups and their automorphism groups, Mem. Amer. Math. Soc. 86 (1990), no. 432, iv-151. MR 90k:20048. Zbl 703.20021.

[10] W. R. Scott, Products of $A_{5}$ and a finite simple group, J. Algebra 37 (1975), no. 1, 165-171. MR 52\#3321. Zbl 317.20012.

[11] C. C. Sims, Computational methods in the study of permutation groups, Computational Problems in Abstract Algebra (Proc. Conf., Oxford, 1967), Pergamon, Oxford, 1970, pp. 169-183. MR 41\#1856. Zbl 215.10002.

[12] M. Suzuki, Group Theory I, Grundlehren der Mathematischen Wissenschaften, vol. 247, Springer-Verlag, Berlin, 1982, translated from the Japanese by the author. MR 82k:20001c. Zbl 472.20001.

[13] G. L. Walls, Groups which are products of finite simple groups, Arch. Math. (Basel) 50 (1988), no. 1, 1-4. MR 88k:20049. Zbl 611.20017.

[14] _ Nonsimple groups which are the product of simple groups, Arch. Math. (Basel) 53 (1989), no. 3, 209-216. MR 90k:20049. Zbl 672.20011.

[15] _ _ Products of simple groups and symmetric groups, Arch. Math. (Basel) 58 (1992), no. 4, 313-321. MR 93a:20032. Zbl 764.20015.

[16] J. Wiegold and A. G. Williamson, The factorisation of the alternating and symmetric groups, Math. Z. 175 (1980), no. 2, 171-179. MR 82a:20008. Zbl 439.20003.

M. R. DARAfsheh: Center for Theoretical Physics and Mathematics, AEOI, P.O. Box 11365-8486, TEHRAN, IRAN

Current address: Department of MATHEMATICS AND COMPUTER SCIENCES, FACUlTy OF SCIENCES, UNIVERSITY OF TEHRAN, TEHRAN, IRAN

E-mail address: daraf@khayam.ut.ac.ir

G. R. ReZAeEzadeh: DePartment of Mathematics, Faculty of Basic SCIENCES, UniveRSITY OF TARBIAT MODDARES, TEHRAN, IRAN

E-mail address: rezaee@net1cs.modares.ac. ir 


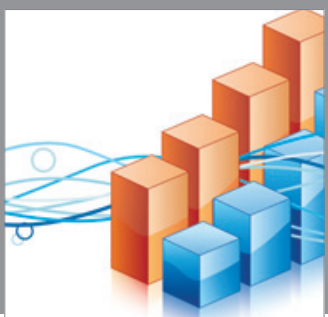

Advances in

Operations Research

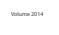

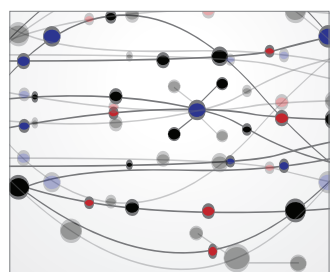

\section{The Scientific} World Journal
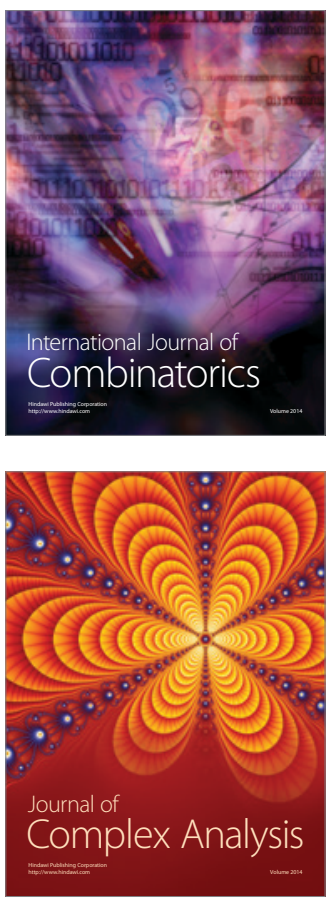

International Journal of

Mathematics and

Mathematical

Sciences
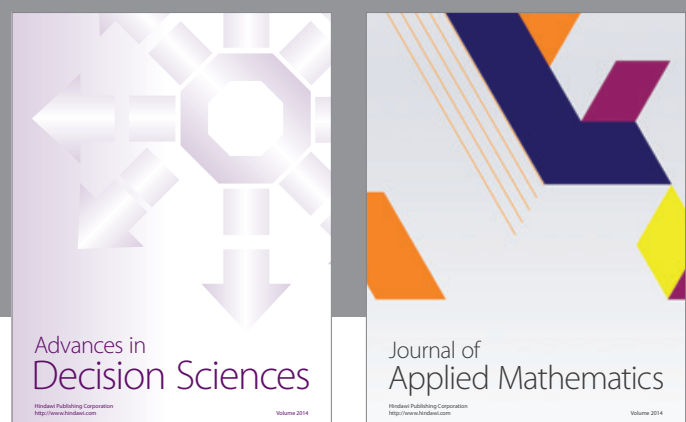

Journal of

Applied Mathematics
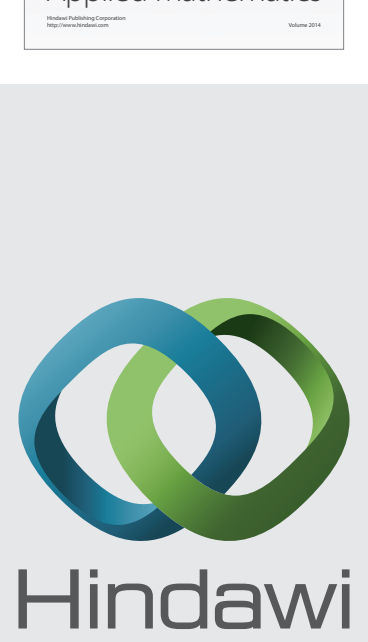

Submit your manuscripts at http://www.hindawi.com
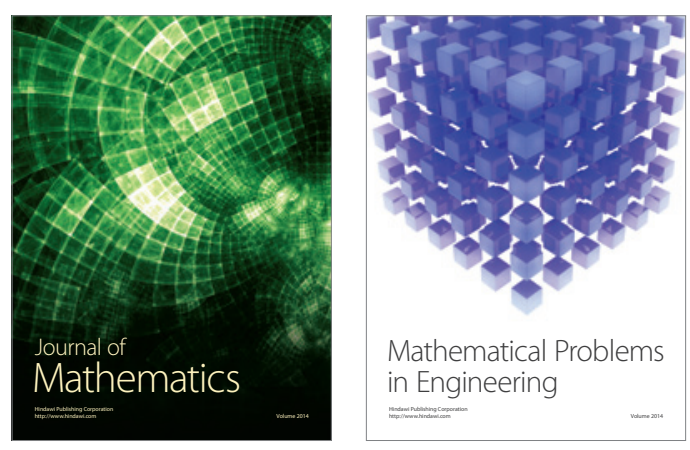

Mathematical Problems in Engineering
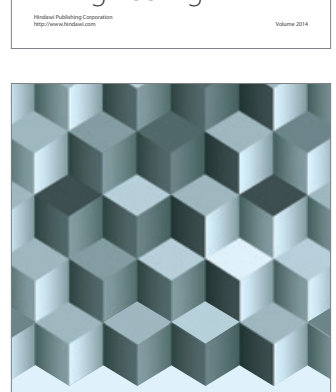

Journal of

Function Spaces
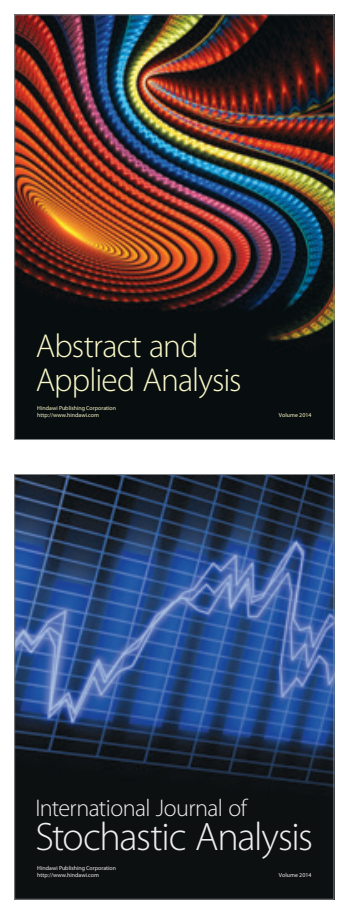

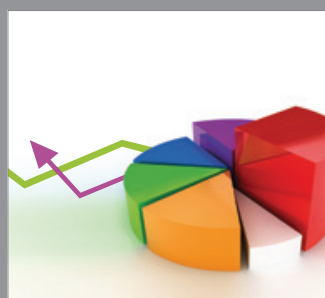

ournal of

Probability and Statistics

Promensencen
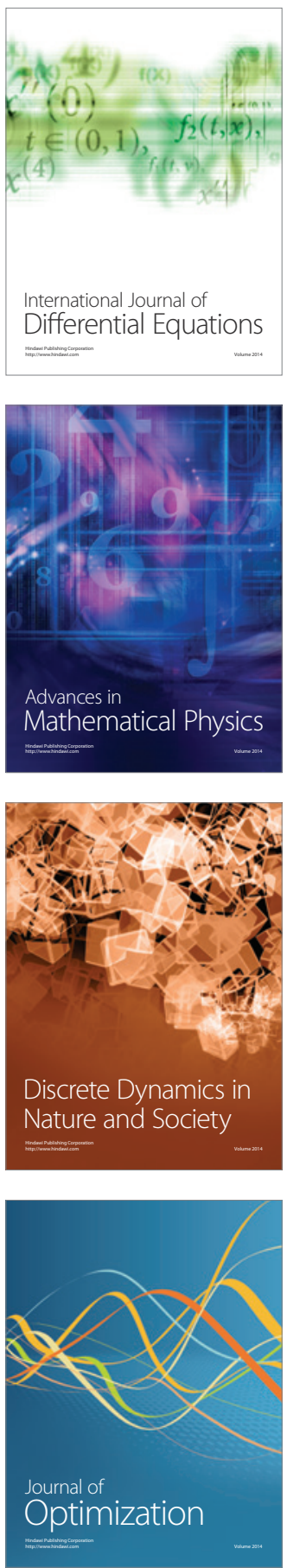